\title{
Incorporating Clinical Decisions into Standardised Caremaps
}

\author{
Scott Mclachlan \\ Queen Mary University of London \\ London, United Kingdom \\ ORCID: 0000-0002-2528-8050
}

\author{
Kudakwashe Dube \\ Massey University \\ Palmerston North, New Zealand \\ ORCID: 0000-0002-2829-8481
}

\author{
Graham A Hitman \\ Queen Mary University of London \\ London, United Kingdom \\ g.a.hitman@qmul.ac.uk
}

\author{
Evangelia Kyrimi \\ Queen Mary University of London \\ London, United Kingdom \\ 0000-0001-6727-2279 \\ Max Marsden \\ Queen Mary University of London \\ London, United Kingdom \\ m.e.r.marsden@qmul.ac.uk \\ Norman E Fenton \\ Queen Mary University of London \\ London, United Kingdom \\ n.fenton@qmul.ac.uk
}

\author{
Bridget J Daley \\ Queen Mary University of London \\ London, United Kingdom \\ ORCID: 0000-0001-8495-3500 \\ Sarah Finer \\ Queen Mary University of London \\ London, United Kingdom \\ s.finer@qmul.ac.uk
}

\begin{abstract}
Caremaps lack a systematic, easy-to-use graphical representation method for care decision points (DPs), which are often missing. This paper addresses this gap by presenting a consistent model and representational notation for clinical decisions. This paper presents: (1) an analysis of clinical decisions that directly impact the path of patient care in the contemporary caremap; (2) an extension of the activity diagram for caremaps; and (3) the extended TaSC (e-TaSC) model and notation for caremaps modelling. Evaluation is done in two case studies: gestational diabetes mellitus and trauma caremaps. In both case studies, e-TaSC enabled systematic consideration and inclusion of clinical DPs leading to clearer, easier-to-follow and more comprehensive caremaps than found in the literature.
\end{abstract}

Keywords—caremaps, clinical decisions, decision points

\section{INTRODUCTION}

The clinical decision-making landscape is becoming increasingly complicated. Patient care involves making complex clinical decisions to determine the next steps of care. Accurate, personalised information enables clinicians to provide precision, rather than just population-based medicine. Medical decision-making is often performed under conditions of uncertainty within a complex decision threshold model. The diagnostic threshold assists the clinician to estimate the probability that the patient has the disease, and evaluate whether further tests are required. The treatment threshold is the point where the probability of disease or its consequences is such that treatment is considered beneficial. This threshold model in caremaps is often represented similar to the if-thenelse rules and multiple cases of condition-action statements similar to the case-switch statement common to software programming languages. To ensure maximum utility, all outcome options within the decision threshold model of a caremap must be represented within the graphical visualisation model. Caremaps like that shown in Fig.4 of Panzarasa et al [1] provide the clinician with a graphical cue representing where a clinical decision needs to be made, while failing to provide justification or necessary criteria to assist them in easily identifying the best path for their patient. Others, like those shown in Fig.1 of Milne et al [2] and Fig.1 of Saint-Jacques et al [3] present quite granular justification for the threshold values or criteria to be considered, but lack any visual cue to easily identify or differentiate Decision Points (DP) from standard activity nodes. This paper contends that integrating DPs into the TaSC caremap model [4] combines: (1) a visual cue for clinicians to easily identify when a clinical decision needs to be made; and (2) a way to identify criteria that enables easy selection of the appropriate treatment path based on accumulated evidence-based knowledge about the current patient.

\section{Clinical Decisions}

Clinical decisions that may give rise to DPs in a caremap result from six aspects of clinical work identified by Richardson et al [5], including: (i) Clinical Evidence: The identification and selection of clinical evidence from clinical trials and clinical practice guidelines for use in the creation of tools, like caremaps, requires decisions regarding how to gather the right clinical findings and interpret them soundly; (ii) Diagnosis: Diagnostic decisions are made regarding the selection and interpretation of diagnostic tests; (iii) Prognosis: Prognosis requires decisions of how to anticipate a given patient's likely course; (iv) Therapy: Therapy decisions consider how to select treatments that do more good than harm; (v) Prevention: Screening and reducing a patient's risk for disease are prevention decisions; and (vi) Education: Consideration of how to teach the clinician, patient or patient's family regarding what is needed fall within the remit of education decisions.

\section{THE E-TASC MODEL}

Previously, we addressed the lack of a standard for caremap structure, content and development process. McLachlan et al [4] proposed TaSC. Presented here, the extended TaSC model is termed e-TaSC. Structure: Caremaps with DPs are presented as flow diagrams. Fig.1 presents the e-TaSC standardised structural model of the caremap with DPs. The elements are inspired by the standardised pictorial elements observed in UML and hard state chart notations. Content: Similar to TaSC, three main content types are captured in e-TaSC; diagnosis, treatment, and management/monitoring. As shown in Fig.1, these broad content types are related to a set of specific medical activities and decisions. Each represents a different caremap level, while the activities and decisions are components of the caremap. Development: The DPs identified for inclusion in a caremap resulted from one of three activities where: (1) a divergence was seen in the path of a caremap - i.e. where two or more paths were already being represented as possible outcomes from an activity node; (2) the clinical practice guideline (CPG) identified a scale or set of diagnostic thresholds for use in deciding whether or which treatment a 
patient should receive; or (3) where the expert clinicians we consulted identified availability of two or more treatments for a given test result, symptomology or diagnostic activity.

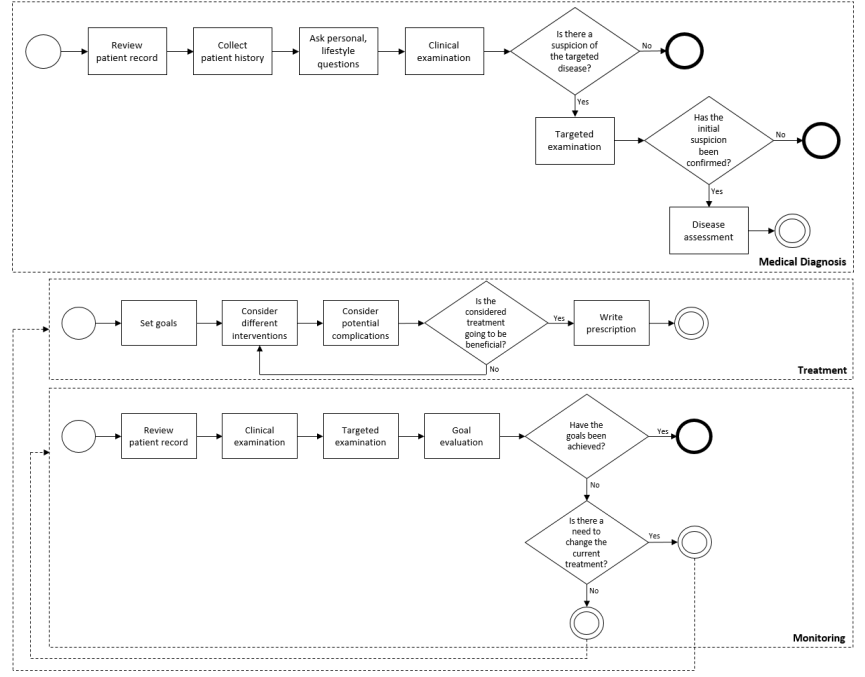

Fig. 1. The e-TaSC content model for the Caremap with decisions

\section{CASE STUdies}

Two case studies were used to evaluate e-TaSC: gestational diabetes mellitus (GDM) and helicopter emergency medical service (HEMS) trauma care. Both are quantitatively evaluated, while the HEMS caremaps was also qualitatively evaluated. In each case we develop caremaps used in development of Bayesian-based clinical decision systems and as part of an approach for elicitation of expert knowledge from clinicians.

\section{A. Case Study 1: GDM Caremap}

Inputs: Inputs were: (a) a clinical practice guideline from Barts Health NHS Trust in East London that is currently in use for the care of women with diabetes in pregnancy; and, (b) clinical expertise and consensus from midwives and diabetologists from the same NHS Trust.

Validation: Validation was performed through consultation seeking consensus from three participating diabetologists with tertiary care experience treating obstetric patients under the CPGs used in the development of the caremaps. The GDM Screening and Diagnosis caremap can be viewed at:

http: //wWw. mclachlandigital. com/GDMdx.png

\section{B. Case Study 2: HEMS Caremaps}

Inputs: Inputs included: (a) a clinical reference textbook prescribed for pre-hospital emergency medical training [47]; (b) clinical practice guidelines issued by national or collegiate health authorities and intended for use by doctors, paramedics and ambulance personnel; (c) current literature on prehospital emergency care; and, (d) clinical expertise from a team of prehospital clinicians, paramedics and trauma fellows. The SCREAMER caremap can be viewed at:

http://www.mclachlandigital.com/screamer.png

The $<\mathrm{C}>\mathrm{ABCDE}$ caremap can be viewed at:

http://www. mclachlandigital.com/cabcde.png

Validation: Initial review and validation was conducted with the clinicians. Once there was consensus, extended validation was performed through reviews of a small group of pre-hospital emergency care clinicians. Some minor modifications and 'fine tuning' was performed on the basis of these reviews that included the addition of clinical factors and symptomatology for a number of decision nodes, and the addition of a process loop for triaging and treatment in situations that presented with multiple casualties.

\section{Evaluation}

Quantitative: As TaSC was refined and became increasingly familiar to those involved, and even as the standard was extended with DPs to become e-TaSC, time and resources to deliver each caremap significantly reduced.

Qualitative: The HEMS caremaps were evaluated through operation of a survey instrument using a forced-choice Likert scale. Survey participants were seven self-identifying experienced emergency and trauma clinicians. In each case clinicians examined SCREAMER and $<\mathrm{C}>\mathrm{ABCDE}$ trauma caremaps to evaluate whether they possessed qualities similar to the CPGs, mnemonic sequence and care processes. Results of the survey demonstrate that caremap structure and path, when examined independently, were considered to be accurate 88\% (Q1) and 76\% (Q2) respectively, while placement, purpose and criterion used to describe DPs were $86 \%(\mathrm{Q} 3)$ and $95 \%$ (Q4) accurate. Caremaps were assessed overall to be $93 \%$ accurate when all elements were examined jointly, and $95 \%$ (Q6) easier to use than clinical documentation they were based on. This survey of practicing clinicians indicates a high degree of accuracy for caremaps developed using the e-TaSC approach. While conclusions cannot be generalised due to the limited number of participants.

\section{SUMMARY}

There has been inconsistency in the way DPs are modelled and incorporated into caremaps. This paper presented the extended TaSC (e-TaSC) model that enables systematic consideration of DPs in a caremap that is being modelled.

\section{ACKNOWLEDGMENTS}

The authors acknowledge support from EPSRC under project EP/P009964/1: PAMBAYESIAN: Patient Managed decision-support using Bayes Networks and Massey University for supporting KD's research work.

\section{REFERENCES}

[1] Panzarasa, S., S. Madde, S. Quaglini, C. Pistarini and M. Stefanelli. (2002). Evidence-based careflow management systems: the case of post-stroke rehabilitation. Journal of biomedical informatics, 35(2), 123-139.

[2] Milne, T., J. Rogers, E. Kinnear, H. Martin, P. Lazzarini, T. Quinton and F. Boyle. (2013). Developing an evidence-based clinical pathway for the assessment, diagnosis and management of acute Charcot NeuroArthropathy: a systematic review. Journal of foot and ankle research, 6(1).

[3] Saint-Jacques, H., V. Burroughs, J. Watkowska, M. Valcarcel, P. Moreno and M. Maw. (2005). Acute coronary syndrome critical pathway: Chest PAIN caremap. A qualitative research study providerlevel intervention. Critical Pathways in Cardiology, 4(3), 145-604.

[4] McLachlan, S., Kyrimi, E., Dube, K., \& Fenton, N. (2019). Clinical caremap development: How can caremaps standardise care when they are not standardised? Proceedings of the $12^{\text {th }}$ International Joint Conference on Biomedical Systems and Technologies (BIOSTEC 2019), volume 5: HEALTHINF, pp 123-134. DOI: $10.5220 / 0007522601230134$.

[5] Richardson, W. S., Wilson, M. C., Nishikawa, J., \& Hayward, R. S. (1995). The well-built clinical question: a key to evidence-based decisions. Acp j club, 123(3) 